\title{
Influence of Building Envelope Type on the Minimum Mechanical Ventilation Rate to Achieve a Positive Indoor Air Pressure
}

\author{
Yuchen $\mathrm{Shi}^{1}$, Xiaofeng $\mathrm{Li}^{1{ }^{1}{ }^{*} \text {, and Seyedehelham Sadatiseyedmahalleh }}{ }^{1}$ \\ ${ }^{1}$ Tsinghua University, Beijing, 100084, China
}

\begin{abstract}
In order to limit the infiltration of outdoor air pollutants, a positive indoor air pressure should be maintained. This study aims to investigate the minimum mechanical ventilation rate to achieve a positive indoor air pressure for large space buildings with different kinds of envelopes. The types of building envelope include envelope with multilayer windows (which generally appears in shopping malls and open-plan office buildings), envelope with entrances on the first floor and multilayer windows, and envelope with openings at bottom and top levels (which generally appears in industrial plants). It is concluded that the minimum ratio between mechanical ventilation and initial infiltration rates to achieve a positive indoor air pressure depends on the window width-height ratio, the vertical spacing between windows, and the number of window layers. More regulations are summarized and analysed to guide the ventilation design.
\end{abstract}

\section{Introduction}

The exposure to indoor air pollutants can negatively affect the health of occupants [1]. One of the main sources of indoor air pollutants is infiltration. This problem is especially serious when outdoor air pollution is severe. Stack effect and wind pressure are two main factors leading to air infiltration in buildings [2]. However, with the rapid construction of high-rise buildings, wind speed in cities has decreased significantly, resulting in horizontal static wind [3]. Horizontal static wind is not conducive to the diffusion and dilution of air pollutants, and is one of the important causes of air pollution [4]. Under the effect of horizontal static wind, the stack effect is the main factor leading to air infiltration in buildings in cities.

In order to continuously protect the building occupants with an acceptable indoor air quality, a positive indoor air pressure should be maintained, that is, the air infiltration rate should be zero [5]. In fact, ASHRAE standard requires mechanically ventilated buildings to maintain a positive indoor air pressure [6]. Based on this rule, some studies have assumed that the infiltration rate is precisely zero when the mechanical ventilating system is operating $[7,8]$. However, many studies have shown that it is difficult to achieve a positive indoor air pressure if the building is air-leaky or the mechanical ventilation rate is low $[9,10]$.

Hence, to create a healthy indoor environment, it is urgent to investigate the effect of mechanical ventilation on infiltration rate under stack effect and the minimum mechanical ventilation rate to achieve a positive indoor air pressure.

Previous studies have focused on this issue for zones with simple crack distributions on the envelopes $[11,12]$.
This study aims to investigate the minimum mechanical ventilation rate to achieve a positive indoor air pressure for buildings with different kinds of envelopes when air infiltration is caused by the stack effect. The types of building envelope include envelope with multilayer windows (which generally appears in shopping malls and open-plan office buildings), envelope with entrances on the first floor and multilayer windows, and envelope with openings at bottom and top levels (which generally appears in industrial plants). A method is put forward to calculate the minimum ratio between mechanical ventilation and initial infiltration rates to achieve a positive indoor air pressure for these buildings. The outcomes are analysed for different types of building envelope.

\section{Method}

Figures 1 and 2 illustrate air infiltration under stack effect when outdoor temperature is higher than indoor temperature (hereinafter, Tout>Tin condition) and when outdoor temperature is lower than indoor temperature (hereinafter, Tout $<$ Tin condition).

In Figures 1 and 2, $Q_{i n f, 0}$ and $Q_{e x f, 0}$ are the initial air infiltration and exfiltration rate when the zone is not mechanically ventilated, $\mathrm{m}^{3} / \mathrm{h} ; Q_{\text {inf }}$ is the air infiltration rate when the zone is mechanically ventilated, $\mathrm{m}^{3} / \mathrm{h} ; Q_{m v}$ is mechanical ventilation rate (i.e., mechanical fresh air rate supplied into the zone), $\mathrm{m}^{3} / \mathrm{h} ; O$ is the initial point which represents the lowest point of the cracks along the vertical direction; $H$ is the height from the initial point to the highest point of the cracks along the vertical direction, $\mathrm{m} ; H_{2}$ and $H_{1}$ are the position of neutral pressure level when the building is mechanically ventilated or not, m;

\footnotetext{
* Corresponding author: xfli@tsinghua.edu.cn
} 
and $A(h)$ is the leakage area distribution function, where $h$ is the height from the initial point to the air leakage position, $\mathrm{m}$.

Figures 1(a) and 2(a) show the initial air infiltration under stack effect when the zone is not mechanically ventilated. In Figures 1(b) and 2(b), when mechanical fresh air is supplied, indoor air is pressurized, and the air pressure profile is shifted to the outside. When the upper end (Tout $>$ Tin condition) or lower end (Tout $<$ Tin condition) of the air pressure profile reaches the building envelope, the infiltration rate is reduced to zero, and the positive indoor air pressure is achieved.

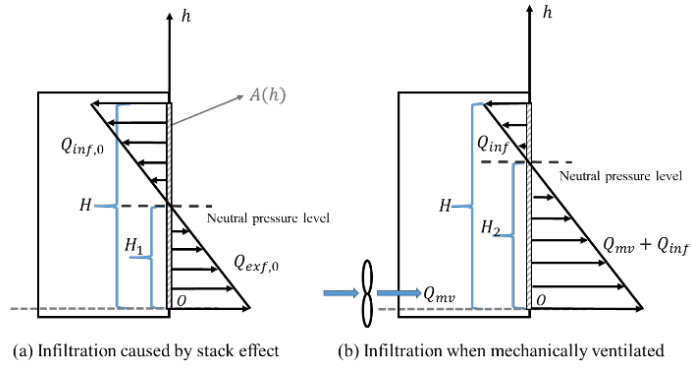

Fig. 1. Pressure profile and air infiltration under stack effect (Tout $>$ Tin condition).

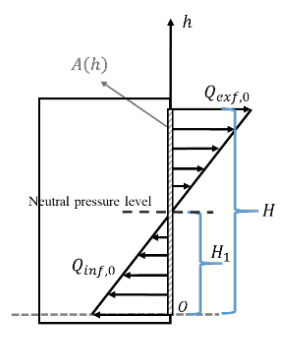

(a) Infiltration caused by stack effect

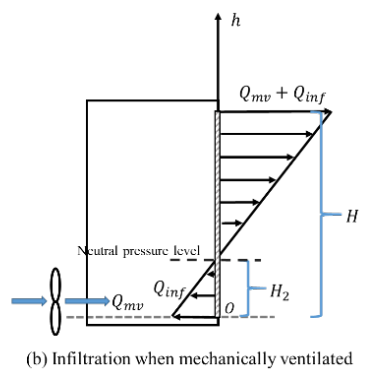

(b) Infiltration when mechanically ventilated
Fig. 2. Pressure profile and air infiltration under stack effect (Tout $<$ Tin condition). cracks [2]:

The method is based on the power law equation of

$$
Q=c A(\Delta p)^{n}
$$

where $Q$ is the air flow rate through cracks, $\mathrm{m}^{3} / \mathrm{h} ; c$ is the air flow coefficient, $\mathrm{m} /\left(\mathrm{h} \cdot \mathrm{Pa}^{\mathrm{n}}\right) ; A$ is the effective leakage area, $\mathrm{m}^{2}$; and $n$ is the pressure exponent, which is commonly set to 0.67 for adventitious cracks [13-15], dimensionless.

Taking the Tout $>$ Tin condition as an example. The excess pressure causing air infiltration in Figure 1(a) is described as

$$
\Delta p=\Delta \rho g\left(h-H_{1}\right)
$$

where $\Delta p$ is the excess pressure at the height of $h, \mathrm{~Pa} ; \rho$ is the difference between indoor and outdoor air densities, $\mathrm{kg} / \mathrm{m}^{3}$; and $g$ is gravity acceleration, $\mathrm{m} / \mathrm{s}^{2}$.

Thus, the initial infiltration rate in Figure 1(a) is

$$
\begin{aligned}
Q_{\text {inf }, 0}= & \int_{H_{1}}^{H} c A(h)\left[\Delta \rho g\left(h-H_{1}\right)\right]^{n} \\
& =\int_{H_{1}}^{H} c f(h)\left[\Delta \rho g\left(h-H_{1}\right)\right]^{n} d h
\end{aligned}
$$

where $f(h)$ is the total width of the cracks on all facades at each height, $\mathrm{m}$; and $d h$ is the differential of $h, \mathrm{~m}$.

Similarly, $Q_{\text {exf }, 0}, Q_{\text {inf }}$, and $Q_{m v}+Q_{\text {inf }}$ in Figure 1 are calculated. Based on the equality between $Q_{\text {inf }, 0}$ and $Q_{\text {exf }, 0}$, the position of neutral pressure level $H_{1}$ is solved. When $Q_{\text {inf }}$ in Figure $1(\mathrm{~b})$ is zero, $H_{2}$ equals $H$, and $Q_{m v}$ reaches the minimum mechanical ventilation rate to achieve a positive indoor air pressure. Define MV ratio as the quotient between mechanical ventilation and initial infiltration rates. Then, the minimum MV ratio to achieve a positive indoor air pressure is

$$
M V_{\text {ratio,min }}=\frac{\int_{0}^{H} f(h)(H-h)^{n} d h}{\int_{H_{1}}^{H} f(h)\left(h-H_{1}\right)^{n} d h}
$$

Based on Eq. (4), when the leakage area distribution function is determined, the minimum MV ratio to achieve a positive indoor air pressure is obtained. Similar equations can be derived for Tout $<$ Tin condition in Fig 2 .

\section{Results}

The minimum MV ratio to achieve a positive indoor air pressure is calculated for buildings with different kinds of envelopes. The types of building envelope include envelope with multilayer windows, envelope with entrances on the first floor and multilayer windows, and envelope with openings at bottom and top levels.

\subsection{Envelope with multilayer windows}

Shopping malls and open-plan office buildings generally have 3 to 10 stories, with an atrium from top to bottom. The surrounding rooms on each floor are opening toward the atrium. The interior space of the building can be regarded as a large single zone with multilayer exterior windows. The crack distribution is illustrated in Figure 3, where $W$ and $L$ are the width and height of each window, $\mathrm{m} ; D$ is the height between windows on adjacent layers, $\mathrm{m} ; m$ is the total number of window layers; and $k$ is the total number of windows in the horizontal direction.

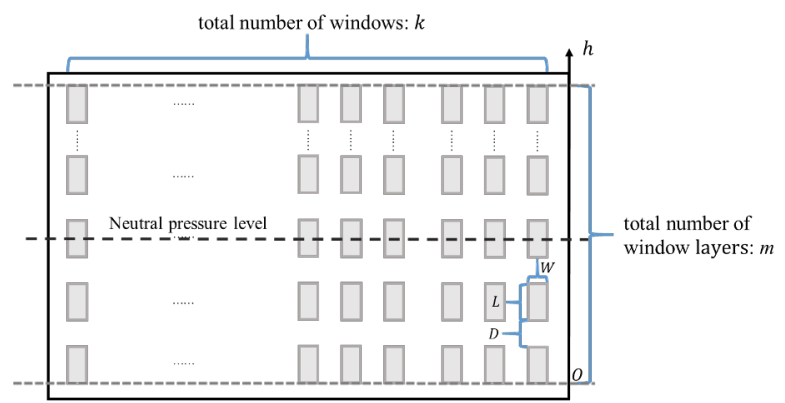

Fig. 3. Crack distribution for a building with multilayer windows.

Air leakage occurs around the openable boundary of the windows. The leakage area on all facades of the building envelope can be described by a piecewise function along the vertical direction. The function is described as 


$$
\begin{aligned}
& A(h)=f(h) d h \\
& =\left\{\begin{aligned}
k W \delta, & h=0, L,(1+\varphi) L,(2+\varphi) L, 2(1+\varphi) L, \ldots,(m-1)(1+\varphi) L,[m+(m-1) \varphi] L \\
2 k \delta d h, & 0<h<L,(1+\varphi) L<h<(2+\varphi) L, \ldots,(m-1)(1+\varphi) L<h<[m+(m-1) \varphi] L \\
0, & L<h<(1+\varphi) L,(2+\varphi) L<h<2(1+\varphi) L, \ldots,[(m-1)+(m-2) \varphi] L<h<(m-1)(1+\varphi) L
\end{aligned}\right.
\end{aligned}
$$

where $\delta$ is the width of the cracks around the exterior windows, which is much smaller compared to the width and height of the windows, $\mathrm{m} ; \varphi$ is the ratio between $D$ and $L$, dimensionless.

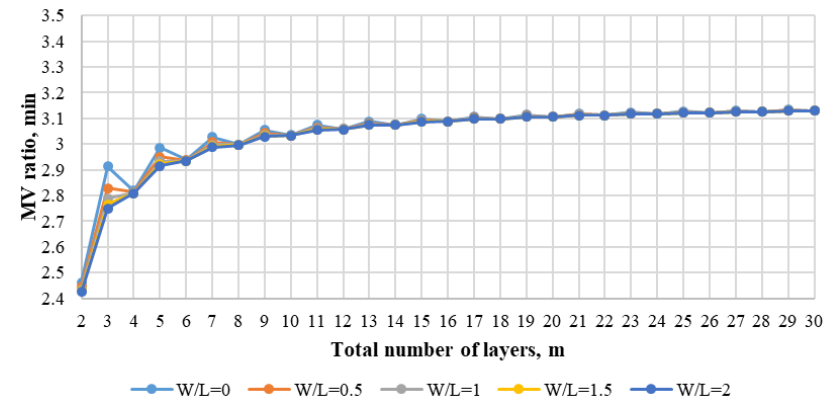

(a) $\mathrm{D} / \mathrm{L}=1$

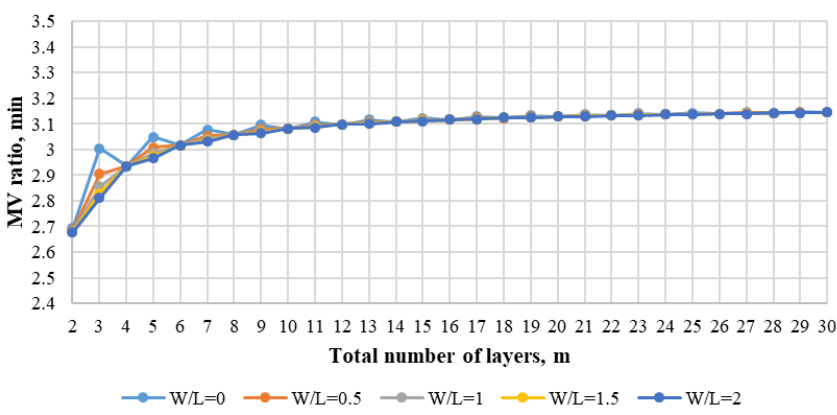

(b) $\mathrm{D} / \mathrm{L}=1 / 2$

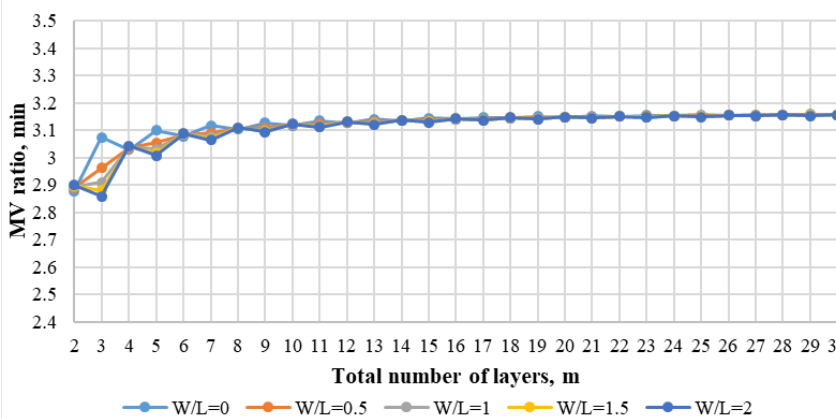

(c) $\mathrm{D} / \mathrm{L}=1 / 4$

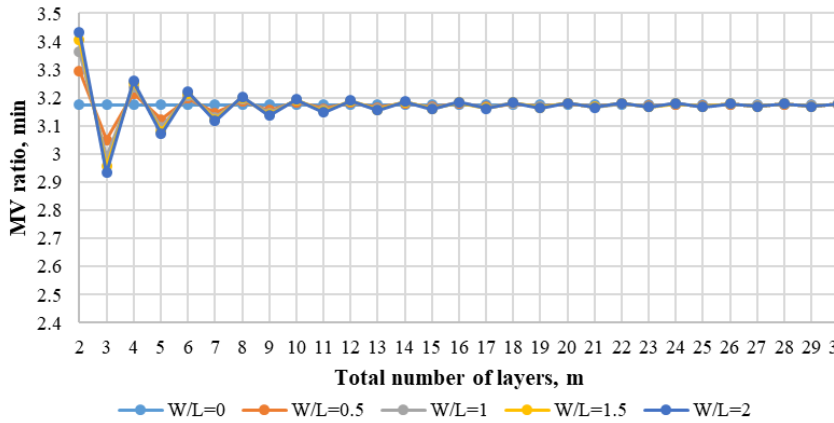

(d) $\mathrm{D} / \mathrm{L}=0$

Fig. 4. Minimum MV ratio for a building with multilayer windows.
In order to cover typical cases, results are analysed for the $\mathrm{W} / \mathrm{L}$ ratio ranging from 0 to $2, \mathrm{D} / \mathrm{L}$ ratio ranging from 0 to 1 , and the number of window layers ranging from 2 to 30, which is illustrated in Figure 4. The outcome shows that the minimum MV ratio to achieve a positive indoor air pressure is related to the width-height ratio of the window (W/L ratio), the height between windows on the adjacent layers (which is presented by $\mathrm{D} / \mathrm{L}$ ratio), and the number of window layers. The outcome also indicates three main regularities:

a) with the number of layers increasing, the minimum MV ratio is almost unaffected by the $\mathrm{W} / \mathrm{L}$ ratio;

b) the minimum MV ratio has different variation laws when the number of layers is odd or even;

c) the limit value of the minimum MV ratio is 3.17 when the number of layers is infinite.

\subsection{Envelope with entrances on the first floor and multilayer windows}

In practice, in the above building with multilayer windows, there could be more openings on the first floor, such as entrances. Suppose that the existence of these entrances makes the leakage area of the first layer $\alpha$ times that of the other layers, then $\alpha$ is the equivalent number of entrances on the first layer. Since the outcome in Figure 4 is unrelated to the total number of windows in the horizontal direction (i.e., $k$ ), the crack distribution for $k=1$ is shown in Figure 5 .

Based on the crack distribution in Figure 5, the leakage area distribution function is obtained. The results calculated by Eq. (4) are illustrated in Figure 6 (only the results when $\alpha$ is 3 and $\mathrm{D} / \mathrm{L}$ is zero are shown for example). As it can be seen, the minimum MV ratio has different variation laws for Tout $>$ Tin and Tout $<$ Tin conditions. Tout $>$ Tin condition has a higher minimum MV ratio than Tout $<$ Tin condition under the same window layers. This is because the neutral pressure level in Figures 1(a) and 2(a) is at the lower middle position of the total crack height. Air infiltrates above the neutral pressure level in Tout $>$ Tin condition and below the neutral pressure level in Tout $<$ Tin condition. Thus, when the positive indoor pressure is achieved, the air pressure profile moves further in Tout $>$ Tin condition than in Tout $<$ Tin condition.

Besides, the three regularities in Section 3.1 are also applicable. 


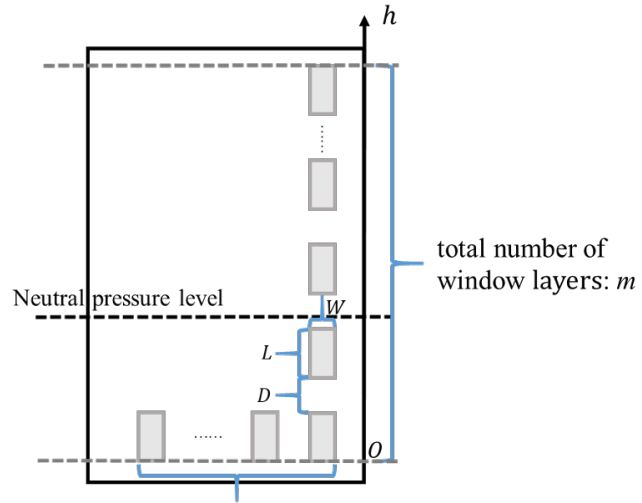

equivalent number of entrances on the first layer: $\alpha$

Fig. 5. Crack distribution for a building with entrances on the first floor and multilayer windows.

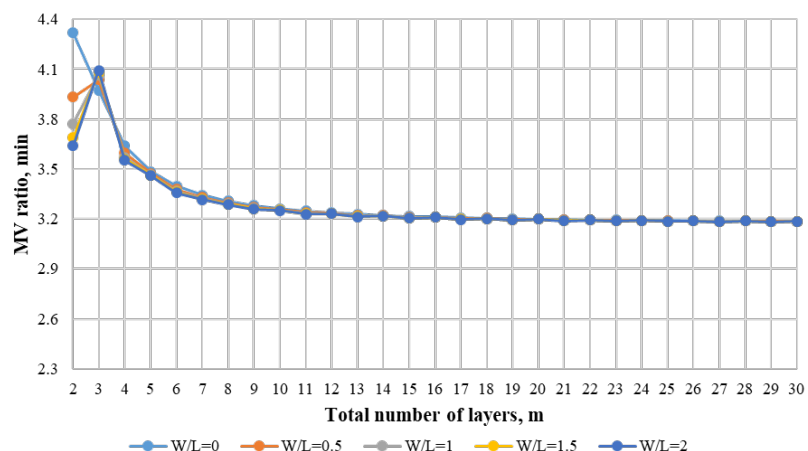

(a) Tout $>$ Tin condition

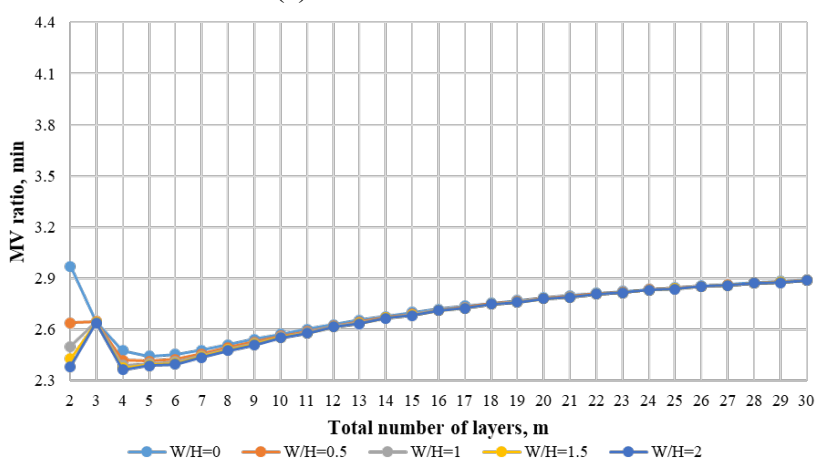

(b) Tout $<$ Tin condition

Fig. 6. Minimum MV ratio for a building with entrances on the first floor and multilayer windows $(\alpha=3, \mathrm{D} / \mathrm{L}=0)$.

\subsection{Envelope with openings at bottom and top levels}

Industrial plants are usually single spaces with entrances on the ground floor and ventilation windows on the upper floor. The crack distribution is shown in Figure 7. The outcome in Figure 8 indicates different regularities from those in Sections 3.1 and 3.2:

a) the minimum MV ratio is almost unaffected by the $\mathrm{W} / \mathrm{L}$ ratio;

b) the minimum MV ratio decreases monotonically with the total height (which is represented by $m$ );

c) the limit value of the minimum MV ratio is 1.59 when the total height is infinite.

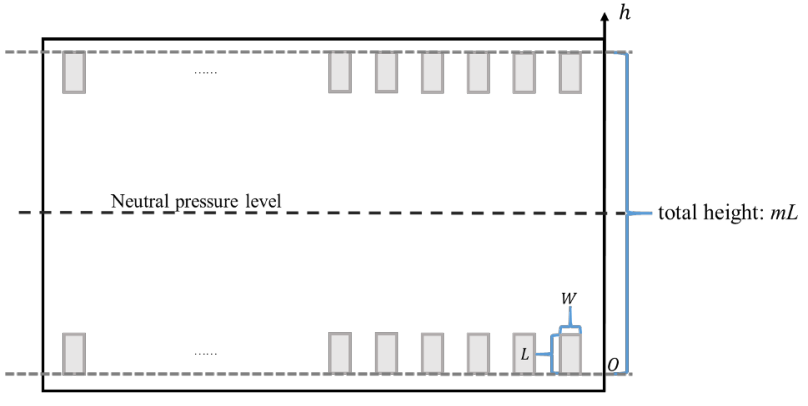

Fig. 7. Crack distribution for a building with openings at bottom and top levels.

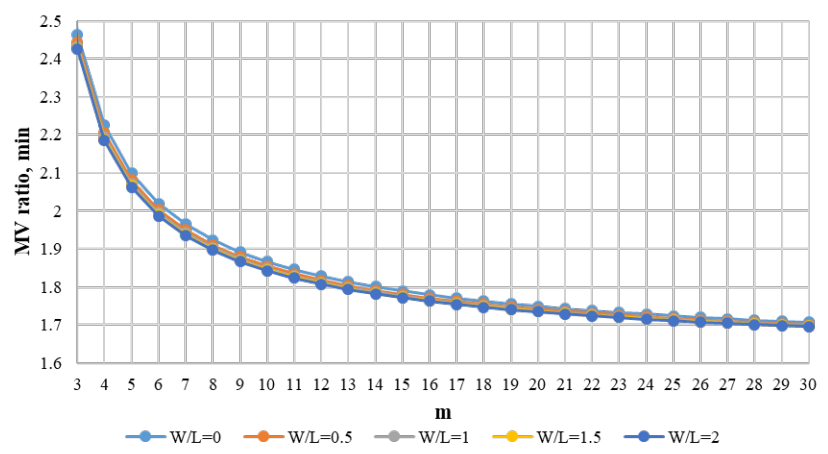

Fig. 8. Minimum MV ratio for a building with openings at bottom and top levels.

\section{Conclusion}

Positive indoor air pressure should be maintained to prevent outdoor air pollutants from infiltrating indoors. Therefore, it is necessary to figure out the minimum mechanical ventilation rate to achieve a positive indoor air pressure. This study focuses on buildings with complex crack distributions on the envelopes and calculates the minimum ratio between mechanical ventilation and initial infiltration rates to achieve a positive indoor air pressure (i.e., the minimum MV ratio) for these buildings.

It is concluded that the minimum MV ratio is almost unaffected by the width-height ratio of the windows. For buildings with multilayer windows (and with entrances on the first floor) which generally appear in shopping malls and open-plan office buildings, the minimum MV ratio has different variation laws when the number of layers is odd or even; the limit value of the minimum MV ratio is 3.17 when the number of layers is infinite. For buildings with openings at bottom and top levels which generally appear in industrial plants, the minimum MV ratio decreases monotonically with the total height; the limit value of the minimum MV ratio is 1.59 when the total height is infinite. Specific law curves are shown in Figures 4,6 , and 8 .

\section{References}

1. J.D. Spengler and K. Sexton, Science, 221, 4605: p. 9-17 (1983) 
2. ASHRAE, Handbook-Fundamentals, American Society of Heating, Refrigerating and Air Conditioning Engineers, Inc (2009)

3. J. Zhou, The Open Cybernetics \& Systemics Journal, 9, p. 1311-1314 (2015)

4. Y. Chen and J. Xiong, The Science Education Article Collects, 4, p. 52 (2014)

5. L.Y. Cooper, Sci. Technol. Built Environ., 24, 10, p. 1114-1140 (2018)

6. ASHRAE, Standard 62.1-2016, American Society for Heating, Refrigeration, and Air-Conditioning Engineers, Inc (2016)

7. J. C. Lam, K. K. Wan, D. Liu, and C. Tsang, Energy Conv. Manag., 51, 12, p. 2692-2697 (2010)
8. H. Ma, Master thesis, Tsinghua University (2016)

9. M.H. Sherman, J.M. Logue, and B.C. Singer, HVAC\&R Res., 17, 2, p. 159-173 (2011)

10. A.K. Persily, Indoor Air, 26, 1, p. 97-111 (2016)

11. G. T. Tamura and C. Y. Shaw, ASHRAE Transactions, 82 (1976)

12. Y. Shi and X. Li, Build. Environ., 131, p. 1-11 (2018)

13. B. Jones, R. Lowe, M. Davies, Z. Chalabi, P. Das, and I. Ridley, Build. Serv. Eng. Res. Technol, 35, 4, p. 408-416 (2014)

14. M. H. Sherman, D. J. Wilson, and D. E. Kiel, ASTM International (1986)

15. I.S. Walker and D.J. Wilson, HVAC\&R Res., 4, 2, p. 119-139 (1998) 